University of Wollongong

Research Online

Faculty of Engineering and Information

Faculty of Engineering and Information

Sciences - Papers: Part B

Sciences

2020

A semi-analytic valuation of American options under a two-state regimeswitching economy

Xiaoping Lu

University of Wollongong, xplu@uow.edu.au

Endah Putri

University of Wollongong, ermp358@uowmail.edu.au

Follow this and additional works at: https://ro.uow.edu.au/eispapers1

Part of the Engineering Commons, and the Science and Technology Studies Commons

Research Online is the open access institutional repository for the University of Wollongong. For further information contact the UOW Library: research-pubs@uow.edu.au 


\title{
A semi-analytic valuation of American options under a two-state regime- switching economy
}

\author{
Abstract \\ In this study, we develop a semi-analytic method to evaluate American options under a two-state regime- \\ switching economy. The two free boundaries corresponding to the states divide the pricing domain into \\ two regions: a common continuation region and a transition region. Non-linear partial differential equation \\ (PDE) systems are derived under the Black-Scholes framework for each region. The Laplace transform \\ method is used to solve the PDE systems. Equations for determining the optimal exercise prices are \\ obtained analytically and solved numerically in the Laplace space. A numerical inversion technique is then \\ used to obtain the free boundaries and the option prices in the original time space. The results of various \\ examples show that our technique is efficient and accurate.

\section{Disciplines} \\ Engineering | Science and Technology Studies

\section{Publication Details} \\ Lu, X. \& Putri, E. R. M. (2020). A semi-analytic valuation of American options under a two-state regime- \\ switching economy. Physica A: Statistical Mechanics and its Applications, 538 122968-1-122968-15.
}




\title{
A semi-analytic valuation of American options under a two-state regime-switching economy
}

\author{
Xiaoping Lu* and Endah R.M. Putri ${ }^{\dagger}$ \\ School of Mathematics and Applied Statistics \\ University of Wollongong, Australia
}

\begin{abstract}
In this study, we develop a semi-analytic method to evaluate American options under a two-state regime-switching economy. The two free boundaries corresponding to the states divide the pricing domain into two regions: a common continuation region and a transition region. Non-linear partial differential equation (PDE) systems are derived under the BlackScholes framework for each region. The Laplace transform method is used to solve the PDE systems. Equations for determining the optimal exercise prices are obtained analytically and solved numerically in the Laplace space. A numerical inversion technique is then used to obtain the free boundaries and the option prices in the original time space. The results of various examples show that our technique is efficient and accurate.
\end{abstract}

Keywords: American options, Regime-switching, Semi-analytic solution, Laplace transform

\section{Introduction}

The classical Black-Scholes model for option pricing assumes constant volatility. However, the volatility, which represents the risk of stock returns [18] and the economic condition [7], does change over time in financial markets [34-36]. In addition, the presence of a volatility smile (or skew) and a volatility term structure shows that the classical Black-Scholes model does not fully reflect market dynamics [27]. Thus, there is a practical need to search for models that better capture market dynamics.

There are various studies in the literature, which aim to provide more realistic reflection of financial markets. The models can be mainly categorized into the following: the volatility being treated as an unknown process, local volatility, stochastic volatility models, and regime-switching models. The approach by letting volatility as an unknown process was proposed by Avellaneda et al. [2]. The introduction of this unknown volatility, however, can lead to an extra non-linearity

\footnotetext{
${ }^{*}$ Corresponding author, xplu@uow.edu.au

${ }^{\dagger}$ Permanent address: Department of Mathematics, Sepuluh Nopember Institute of Technology, Indonesia
} 
to the PDE which increases the difficulty in the solution process as pointed out in [3]. Local volatility as a deterministic function of stock price and time is proposed by Dupire, Derman and Kani $[15,16]$. This volatility model has been widely used in industry for its fast calibration [17]. However, the model needs continuous and smooth implied volatility surface, and numerical method applied for local volatility can be unstable. Some studies also pointed out that the performance of local volatility model is not satisfying since it is too restrictive [8, 32].

The stochastic model is considered to be more realistic [1]. Pricing models of a European option with stochastic volatility are used extensively in the literature, such as $[4,13,33,39]$. Hull and White [26] proposed a model which used a log-normal distribution in their study. Stein and Stein [38] proposed the use of Ornstein-Uhlenbeck process in the pricing process. An analytical solution of European option with stochastic volatility in integral form is found by Heston [23]. Extension studies to deal with the integral form of Heston's solution is proposed by Zhu and Chen [48] and Zhylyevskyy [50]. However, numerical or approximation methods are still preferred over the more complicated closed form solutions due to the difficulties in numerical calculation in the Heston form solutions [12, 28].

Recently, regime-switching models have drawn much attention for option valuation $[14,20$, $21,25,41,43,44]$. In a regime-switching model, a key parameter of an asset, volatility, is driven by the market regime. In reality, the market change is driven by economic changes, investor's mood, business cycle expansion, etc. which often shows cyclic patterns [5]. The market regime switches among a finite number of states which represent the financial market conditions. A good agreement between a regime-switching model and empirical data in modeling the economic changes is reported in the study by Hardy [22]. Furthermore, the regime-switching models are less computation-intensive compared to the stochastic volatility models [25, 45].

While European options with regime-switching have been solved analytically in [11, 30, 31, 47], there is no analytical solution for the American counterpart due to the presence of the freeboundaries, except for perpetual options as presented by Guo in [20]. Numerical and analytical approximation methods have been developed as alternatives since the analytic solution for the American options with regime-switching is extremely difficult or even impossible to obtain. Numerical methods to value American options with regime-switching include, among others, the penalty method [29], finite element method [24, 40], and tree method [44]. To the best of the authors' knowledge, the only analytical approximation method available in the literature is the valuation of American options with regime-switching in finite maturity by Buffington and Elliott [10]. Their valuation is based on risk-neutral pricing, and an extension of the quadratic approximation of Barone-Adesi-Whaley [6]. The price of an American option with regime switching is decomposed into a European option part and an extra part as a result of American style privilege. While it is great for showing the American-style effect, the implementation of the method in [10] is not straight-forward. One should first calculate the European option value with regime switching, the calculation of which is not simple because although the solution is in closed form, it is in double integral form as the result of the discounted expectation (see the detail in $[10,19]$ ). In 
addition, there are two non-linear coupled equations for the optimal exercise prices to be solved numerically before the values of the option can be calculated.

In this work, we develop a semi-analytical method for the pricing of American options in a two-state regime-switching economy under the Black-Scholes framework. We aim to provide a method which is both efficient and accurate. Our method is based on the semi-analytic method in Zhu [46], where a pseudo-steady state approximation in Laplace space is used to tackle the free boundary in finite maturity American option problems. We follow Buffington and Elliott [10] and Guo $[20,21]$ in constructing the pricing domain for setting up the coupled partial differential equations for the different states. The equations for calculating the optimal exercise prices and option values are obtained in Laplace space analytically. The optimal boundaries are solved numerically in the Laplace space, then the results are inverted back to the original time space by the Stehfest method [37]. Although we cannot compare our results directly with those in [10] due to that fact that their numerical results are unavailable, we believe that our method should be more efficient judging by both solution processes and our computation times. As mentioned above the value of the American option in [10] is based on its European counterpart so in terms of computation one goes through the processes of calculating the European prices which involve double integrals and then the extra part due to American-style exercise right. In principal, our method without the need of evaluating double integrals would enhance computational efficiency. Our easy to implement approach coupled with its well balanced efficiency and accuracy makes it more advantageous and useful for practical applications.

The rest of the paper is organized as follows. Section 2 presents the formulation of the partial differential equation (PDE) systems for the pricing of American options under a regime-switching economy. Section 3 discusses the solution procedures of the PDE systems. Numerical examples are presented in Section 4 to show the accuracy and efficiency of the proposed method. Concluding remarks are given in Section 5.

\section{Formulation of the PDE system}

In a regime-switching economy, volatility, a key parameter of an asset is driven by market regimes which switch among a finite number of states representing the changes in financial markets. The stock price dynamics follows the SDE:

$$
d S_{t}=(\mu-\delta) S_{t} d t+\sigma_{\bar{\varepsilon}(t)} S_{t} d W_{t}
$$

where $S_{t}$ is the underlying asset, $\mu$ is the drift, $\delta$ is a continuous dividend paid, $\bar{\varepsilon}(t)$ is a stochastic process representing the state of the economic cycle, and $W_{t}$ is a Wiener process independent of $\bar{\varepsilon}(t)$.

It is assumed that $\bar{\varepsilon}(t)$ has finite state space $i, j \in I$ where $i \neq j$ and satisfies the Markov property. The volatility $\sigma_{\bar{\varepsilon}(t)}$ now is a stochastic process and has a different value for different 
states $\bar{\varepsilon}(t)$. In this work, we assume that there are two regimes in the market representing two basic economic cycles

$$
\bar{\varepsilon}(t)= \begin{cases}1, & \text { the economy in a state of growth } \\ 2, & \text { the economy in a state of recession }\end{cases}
$$

The transition between states occurs as a Poisson process and is defined as follows,

$$
P(N(t+\tau)-N(t)=N(\tau)=k)=\frac{\left(\lambda_{i j} \tau\right)^{k} e^{-\lambda_{i j} \tau}}{k !},\{i, j=1,2, i \neq j\},\{k=0,1,2, \ldots\}
$$

where $\lambda_{i j}>0$ is the rate of the jump from state $i$ to state $j$, and $k$ is the number of occurrences of the jump within the time interval $\tau$.

The presence of a regime-switching implies that the market is incomplete and the risk associated with the regime-switching cannot be uniquely determined and inseparable. Naik [31] shows that separable risk can be easily turned into inseparable one by adjusting the rate parameter of the switching process, without a loss of generality. Therefore, in this paper we assume that the risk is separable and not priced as in the works by Zhu et. al [47], Naik [31], Boyle [9] and Yi [42]. Furthermore, we assume the risk-free interest rate $r$ remains constant.

Let the value of an American option when the economy is in the state of growth $(\bar{\varepsilon}(t)=1)$ be $V_{1}$ with volatility $\sigma_{1}$. Then $V_{2}$ represents the value of the option when the economy condition is in a state of recession $(\bar{\varepsilon}(t)=2)$ with volatility $\sigma_{2}$. We construct a portfolio by purchasing an option $V$ and short $\Delta$ amount of underlying asset $S$,

$$
\Pi=V-\Delta S
$$

Consider at a time $t$ when $\bar{\varepsilon}(t)=1$, the value of the option would be $V_{1}(S, t)$. After a short time interval $d t$, the change in the portfolio is $d \Pi=d V-\Delta d S$. However, $d V=d V_{1}$ with the probability of $1-\lambda_{12} d t$, and $d V=V_{2}-V_{1}+d V_{2}$ with the probability of $\lambda_{12} d t$. Under risk-neutral valuation, the investor is expected to make as much profit from the portfolio as they would from a risk-free investment, that is, $\mathbb{E}[d \Pi]=r \Pi d t$, where $r$ is the risk-free interest rate. Using Ito's lemma and equation (2.1), the following pricing PDE for $V_{1}(S, t)$ can be derived (cf. [10], [47])

$$
\frac{\partial V_{1}}{\partial t}+\frac{1}{2} \sigma_{1}^{2} S^{2} \frac{\partial^{2} V_{1}}{\partial S^{2}}+(r-\delta) S \frac{\partial V_{1}}{\partial S}-r V_{1}=\lambda_{12}\left(V_{1}-V_{2}\right)
$$

Similarly the PDE for $V_{2}(S, t)$ is derived as

$$
\frac{\partial V_{2}}{\partial t}+\frac{1}{2} \sigma_{2}^{2} S^{2} \frac{\partial^{2} V_{2}}{\partial S^{2}}+(r-\delta) S \frac{\partial V_{2}}{\partial S}-r V_{2}=\lambda_{21}\left(V_{2}-V_{1}\right)
$$

In the following, the formulation of American calls under a regime-switching economy is presented. The formulation for American puts with regime-switching can be carried out in the 
same way, or the values could be recovered from the put-call symmetry for American options [40]:

$$
\begin{gathered}
S_{f i}^{P}(t ; r ; \delta) S_{f i}^{C}(t ; \delta ; r)=K^{2}, 0 \leq t \leq T \\
P_{i}(S, t ; r ; \delta)=\frac{S}{K} C_{i}\left(K^{2} / S, t ; \delta ; r\right), 0 \leq t \leq T, 0<S
\end{gathered}
$$

where $K$ is the strike price, $T$ the expiry date, $P_{i}$ and $C_{i}$ are the put and call prices, $S_{f i}$ stands for the optimal exercise price, and $S_{f i}^{k}(k=P, C)$ optimal exercise price for put or call, respectively, corresponding to the economy states $i=1,2$.

American options under a two-state regime-switching economy have two optimal exercise boundaries, $S_{f 1}$ and $S_{f 2}$ corresponding to $\sigma_{1}$ and $\sigma_{2}$, respectively. On the assumption in Equation (2.2), $\sigma_{2}<\sigma_{1}$ so $S_{f 2}<S_{f 1}$ for American calls. The optimal boundaries $S_{f 1}$ and $S_{f 2}$ divide the pricing domain into two regions: a common continuation region and a transition region as discussed in the work of Buffington and Elliott [10]. As a result, the stock price, $S$, is in the common continuation region if $0 \leq S \leq S_{f 2}$, and in the transition region if $S_{f 2} \leq S \leq S_{f 1}$ as shown in Figure 2.1. The PDE system for each region is presented in the following subsections.

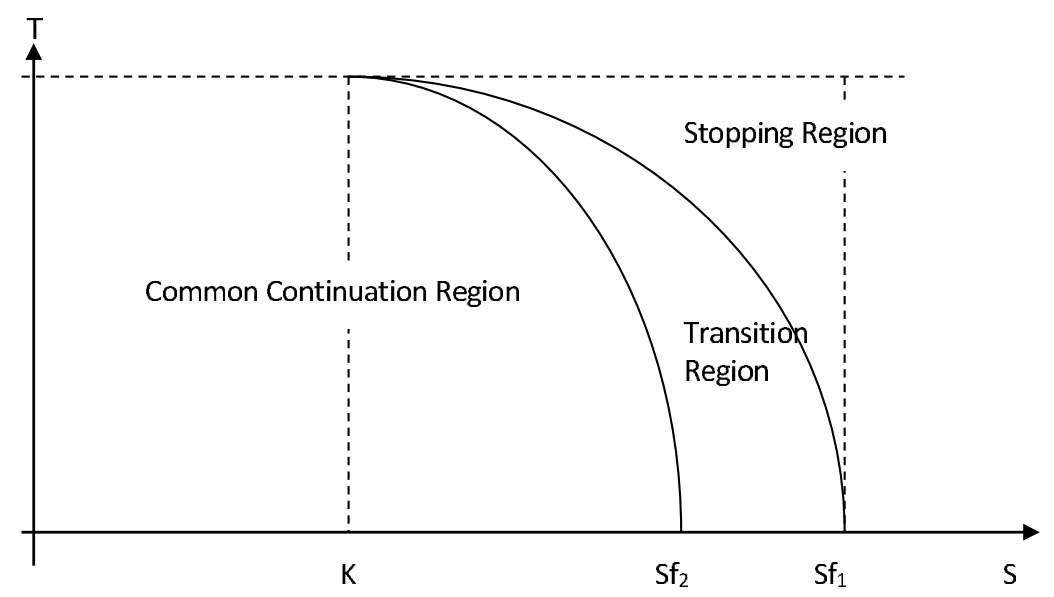

Figure 2.1: The option pricing domain with two optimal exercise boundaries

\subsection{Common Continuation Region}

In the common continuation region $\left(0 \leq S \leq S_{f 2}\right)$, there are two coupled equations governing option values $V_{1}$ and $V_{2}$ :

$$
\left\{\begin{array}{l}
\frac{\partial V_{1}}{\partial t}+\frac{1}{2} \sigma_{1}^{2} S^{2} \frac{\partial^{2} V_{1}}{\partial S^{2}}+(r-\delta) S \frac{\partial V_{1}}{\partial S}-r V_{1}=\lambda_{12}\left(V_{1}-V_{2}\right) \\
\frac{\partial V_{2}}{\partial t}+\frac{1}{2} \sigma_{2}^{2} S^{2} \frac{\partial^{2} V_{2}}{\partial S^{2}}+(r-\delta) S \frac{\partial V_{2}}{\partial S}-r V_{2}=\lambda_{21}\left(V_{2}-V_{1}\right)
\end{array}\right.
$$

An option will be worthless when the stock price is zero. At the end of the contract, the value 
of an option should be equal to its payoff function. Therefore, we have

$$
\begin{gathered}
V_{i}(0, t)=0 \\
V_{i}(S, T)=\max (S-K, 0)
\end{gathered}
$$

The optimal exercise price $S_{f 1}$ is outside of the common continuation region, so equations (2.5) and (2.6) are the only conditions for option value $V_{1}$.

The optimal exercise price $S_{f 2}$ bounds the common continuation region. Thus, at $S=S_{f 2}(t)$, $V_{2}$ needs to satisfy the following boundary conditions:

$$
\left\{\begin{array}{l}
V_{2}\left(S_{f 2}, t\right)=S_{f 2}(t)-K \\
\frac{\partial V_{2}}{\partial S}\left(S_{f 2}, t\right)=1
\end{array}\right.
$$

\subsection{Transition Region}

In the transition region, $S_{f 2} \leq S \leq S_{f 1}$, the value of $V_{2}$ is just the intrinsic value, that is, $V_{2}=S-K$. Thus, the PDE system for this region contains only $V_{1}$ as an unknown function.

$$
\left\{\begin{array}{l}
\frac{\partial V_{1}}{\partial t}+\frac{1}{2} \sigma_{1}^{2} S^{2} \frac{\partial^{2} V_{1}}{\partial S^{2}}+(r-\delta) S \frac{\partial V_{1}}{\partial S}-r V_{1}=\lambda_{12}\left(V_{1}-(S-K)\right) \\
V_{1}(S, T)=\max (S-K, 0) \\
V_{1}\left(S_{f 1}, t\right)=S_{f 1}-K \\
\frac{\partial V_{1}}{\partial S}\left(S_{f 1}, t\right)=1
\end{array}\right.
$$

Note that the terminal condition $V_{1}(S, T)=S-K$, since in the transition region $S>K$.

\subsection{Continuity Condition}

Since the pricing domain for $V_{1}$ consists of two regions, it is necessary to assure the continuity and smoothness of $V_{1}$ at the intersection, that is, at $S=S_{f 2}$. Therefore,

$$
\begin{aligned}
\lim _{S \rightarrow S_{f 2}^{-}} V_{1} & =\lim _{S \rightarrow S_{f 2}^{+}} V_{1} \\
\lim _{S \rightarrow S_{f 2}^{-}} \frac{\partial V_{1}}{\partial S} & =\lim _{S \rightarrow S_{f 2}^{+}} \frac{\partial V_{1}}{\partial S}
\end{aligned}
$$

Equations (2.4)-(2.9) form the complete PDE system for the pricing of an American call under a two-state regime-switching economy. We will discuss the solution procedures in the following section. 


\section{Our Solution Procedure}

Before we start to solve the PDE systems, the following change of variables is introduced:

$$
X=\frac{S}{K}, \tau=T-t, U_{i}=\frac{V_{i}}{K}, \quad i=1,2
$$

The resulting PDE systems in the common continuation region are:

$$
\left\{\begin{array}{l}
-\frac{\partial U_{1}}{\partial \tau}+\frac{1}{2} \sigma_{1}^{2} X^{2} \frac{\partial^{2} U_{1}}{\partial X^{2}}+(r-\delta) X \frac{\partial U_{1}}{\partial X}-r U_{1}=\lambda_{12}\left(U_{1}-U_{2}\right) \\
U_{1}(0, \tau)=0 \\
U_{1}(X, 0)=\max (X-1,0) \\
-\frac{\partial U_{2}}{\partial \tau}+\frac{1}{2} \sigma_{2}^{2} X^{2} \frac{\partial^{2} U_{2}}{\partial X^{2}}+(r-\delta) X \frac{\partial U_{2}}{\partial X}-r U_{2}=\lambda_{21}\left(U_{2}-U_{1}\right) \\
U_{2}(0, \tau)=0 \\
U_{2}(X, 0)=\max (X-1,0) \\
U_{2}\left(X_{f 2}, \tau\right)=X_{f 2}-1 \\
\frac{\partial U_{2}}{\partial X}\left(X_{f 2}, \tau\right)=1
\end{array}\right.
$$

where $\tau \in[0, T], X \in\left[0, X_{f 2}\right]$.

The PDE system in the transition region is:

$$
\left\{\begin{array}{l}
-\frac{\partial U_{1}}{\partial \tau}+\frac{1}{2} \sigma_{1}^{2} X^{2} \frac{\partial^{2} U_{1}}{\partial X^{2}}+(r-\delta) X \frac{\partial U_{1}}{\partial X}-r U_{1}=\lambda_{12}\left[U_{1}-(X-1)\right] \\
U_{1}(X, 0)=X-1 \\
U_{1}\left(X_{f 1}, \tau\right)=X_{f 1}-1 \\
\frac{\partial U_{1}}{\partial X}\left(X_{f 1}, \tau\right)=1
\end{array}\right.
$$

where $\tau \in[0, T], X \in\left[X_{f 2}, X_{f 1}\right]$.

The continuity conditions become:

$$
\begin{gathered}
\lim _{X \rightarrow X_{f 2}^{-}} U_{1}=\lim _{X \rightarrow X_{f 2}^{+}} U_{1} \\
\lim _{X \rightarrow X_{f 2}^{-}} \frac{\partial U_{1}}{\partial X}=\lim _{X \rightarrow X_{f 2}^{+}} \frac{\partial U_{1}}{\partial X}
\end{gathered}
$$

We apply the Laplace transform method to solve the PDE systems in (3.1) and (3.2) with the conditions in (3.3). The pricing PDE systems are transformed into a set of ODE systems in the Laplace space. Zhu's approach in [46] for the treatment of the free boundary or optimal exercise price in the Laplace space is adopted, that is, the moving boundary $X_{i}=X_{f i}(\tau)$ is replaced by 
$X_{i}=p \bar{X}_{f i}(p)$ in the Laplace space, where

$$
\bar{X}_{f i}(p)=\int_{0}^{\infty} X_{f i}(\tau) e^{-p \tau} d \tau, \quad i=1,2 .
$$

The solutions of the ODE system as the result of the Laplace transform of the PDE system (3.1) are (see Appendix A for detail):

$$
\begin{aligned}
& \bar{U}_{1}(X, p)= \begin{cases}A_{1} X^{k_{1}}+A_{2} X^{k_{2}}+A_{3} X^{k_{3}}+A_{4} X^{k_{4}}+\phi(X, p), & \text { if } 1<X \leq X_{f 2} ; \\
A_{5} X^{k_{3}}+A_{6} X^{k_{4}}, & \text { if } 0 \leq X \leq 1 ;\end{cases} \\
& \bar{U}_{2}(X, p)= \begin{cases}B_{1} X^{k_{1}}+B_{2} X^{k_{2}}+B_{3} X^{k_{3}}+B_{4} X^{k_{4}}+\phi(X, p), & \text { if } 1<X \leq X_{f 2} ; \\
B_{5} X^{k_{3}}+B_{6} X^{k_{4}}, & \text { if } 0 \leq X \leq 1 ;\end{cases}
\end{aligned}
$$

where

$$
\phi(X, p)=\frac{1}{p+\delta} X-\frac{1}{p+r},
$$

$k_{1}<k_{2}<0<k_{3}<k_{4}$ are the roots of the quartic indicial equation for the ODE system, $A_{i}$ and $B_{i}, i=1,2,3 \cdots 6$, are some constants in the Laplace space. Note that $A_{i}$ and $B_{i}$ are related so only $A_{i}$ appear in the final equations. The details for finding the quartic roots, and the constants are presented in Appendix C.

The solution of the ODE in the Laplace space corresponding to the PDE system (3.2) in the transition region is (detail in Appendix B):

$$
\bar{U}_{1}(X, p)=m_{1} X^{\gamma_{1}}+m_{2} X^{\gamma_{2}}+\psi_{1} X-\psi_{2}, \quad X_{f 2} \leq X \leq X_{f 1}
$$

where

$$
\psi_{1}=\frac{p+\lambda_{12}}{p\left(p+\delta+\lambda_{12}\right)}, \quad \psi_{2}=\frac{p+\lambda_{12}}{p\left(p+r+\lambda_{12}\right)},
$$

$m_{1}$ and $m_{2}$ are integration constants, and $\gamma_{1}$ and $\gamma_{2}$ are the solutions to the indicial equation:

$$
\frac{1}{2} \sigma_{1}^{2} \gamma^{2}+\left(r-\delta-\frac{1}{2} \sigma_{1}^{2}\right) \gamma-\left(p+r+\lambda_{12}\right)=0 .
$$

Applying the necessary boundary conditions and continuity conditions to solutions (3.4), (3.5), 
and (3.6), we obtain the following equations

$$
\begin{aligned}
& \left\{\begin{array}{l}
A_{1}+A_{2}+A_{3}+A_{4}+\frac{1}{p+\delta}-\frac{1}{p+r}=A_{5}+A_{6} \\
A_{1} k_{1}+A_{2} k_{2}+A_{3} k_{3}+A_{4} k_{4}+\frac{1}{p+\delta}=A_{5} k_{3}+A_{6} k_{4} \\
A_{1} l_{1}+A_{2} l_{2}+A_{3} l_{3}+A_{4} l_{4}+\frac{1}{p+\delta}-\frac{1}{p+r}=A_{5} l_{3}+A_{6} l_{4} \\
A_{1} l_{1} k_{1}+A_{2} l_{2} k_{2}+A_{3} l_{3} k_{3}+A_{4} l_{4} k_{4}+\frac{1}{p+\delta}=A_{5} l_{3} k_{3}+A_{6} l_{4} k_{4}
\end{array}\right. \\
& \left\{\begin{array}{l}
A_{1} l_{1}\left(p \bar{X}_{f 2}\right)^{k_{1}}+A_{2} l_{2}\left(p \bar{X}_{f 2}\right)^{k_{2}}+A_{3} l_{3}\left(p \bar{X}_{f 2}\right)^{k_{3}}+A_{4} l_{4}\left(p \bar{X}_{f 2}\right)^{k_{4}}+\frac{1}{p+\delta}\left(p \bar{X}_{f 2}\right)-\frac{1}{p+r} \\
=\frac{1}{p}\left(p \bar{X}_{f 2}\right)-\frac{1}{p} \\
A_{1} l_{1} k_{1}\left(p \bar{X}_{f 2}\right)^{k_{1}-1}+A_{2} l_{2} k_{2}\left(p \bar{X}_{f 2}\right)^{k_{2}-1}+A_{3} l_{3} k_{3}\left(p \bar{X}_{f 2}\right)^{k_{3}-1}+A_{4} l_{4} k_{4}\left(p \bar{X}_{f 2}\right)^{k_{4}-1}+\frac{1}{p+\delta} \\
=\frac{1}{p}
\end{array}\right. \\
& \left\{\begin{array}{l}
m_{1}\left(p \bar{X}_{f 1}\right)^{\gamma_{1}}+m_{2}\left(p \bar{X}_{f 1}\right)^{\gamma_{2}}+\psi_{1}\left(p \bar{X}_{f 1}\right)-\psi_{2}=\frac{1}{p}\left(p \bar{X}_{f 1}\right)-\frac{1}{p} \\
m_{1} \gamma_{1}\left(p \bar{X}_{f 1}\right)^{\gamma_{1}-1}+m_{2} \gamma_{2}\left(p \bar{X}_{f 1}\right)^{\gamma_{2}-1}+\psi_{1}=\frac{1}{p}
\end{array}\right. \\
& \left\{\begin{array}{l}
A_{1}\left(p \bar{X}_{f 2}\right)^{k_{1}}+A_{2}\left(p \bar{X}_{f 2}\right)^{k_{2}}+A_{3}\left(p \bar{X}_{f 2}\right)^{k_{3}}+A_{4}\left(p \bar{X}_{f 2}\right)^{k_{4}}+\frac{1}{p+\delta}\left(p \bar{X}_{f 2}\right)-\frac{1}{p+r} \\
=m_{1}\left(p \bar{X}_{f 2}\right)^{\gamma_{1}}+m_{2}\left(p \bar{X}_{f 2}\right)^{\gamma_{2}}+\psi_{1}\left(p \bar{X}_{f 2}\right)-\psi_{2} \\
A_{1} k_{1}\left(p \bar{X}_{f 2}\right)^{k_{1}-1}+A_{2} k_{2}\left(p \bar{X}_{f 2}\right)^{k_{2}-1}+A_{3} k_{3}\left(p \bar{X}_{f 2}\right)^{k_{3}-1}+A_{4} k_{4}\left(p \bar{X}_{f 2}\right)^{k_{4}-1}+\frac{1}{p+\delta} \\
=m_{1} \gamma_{1}\left(p \bar{X}_{f 2}\right)^{\gamma_{1}-1}+m_{2} \gamma_{2}\left(p \bar{X}_{f 2}\right)^{\gamma_{2}-1}+\psi_{1}
\end{array}\right.
\end{aligned}
$$

Equations $(3.7-3.10)$ consist of 8 unknown constants and 2 unknown dimensionless optimal exercise prices, $\bar{X}_{f 1}$ and $\bar{X}_{f 2}$. It would be extremely difficult and time consuming to solve the 10 equations simultaneously due to the high non-linearity of the terms involving $\bar{X}_{f 1}$ and $\bar{X}_{f 2}$. Instead, we solve the equations 'by parts', that is, in blocks to obtain all constants and eventually the equations for solving $\bar{X}_{f 1}$ and $\bar{X}_{f 2}$.

Because the algebraic manipulations are quite tedious, here we only outline the solution procedures and list all constants in Appendix D.

- Since Equation system (3.7) is independent of $\bar{X}_{f 1}$ and $\bar{X}_{f 2}$, it is solved first to obtain $A_{1}$, $A_{2}, A_{3}-A_{5}$ and $A_{4}-A_{6}$. 
- $A_{3}$ and $A_{4}$ are then obtained in terms of $\bar{X}_{f 2}$ by substituting the now known $A_{1}$ and $A_{2}$ in Equation system (3.8).

- Once $A_{3}$ and $A_{4}$ are solved, $A_{5}$ and $A_{6}$ will follow.

- $m_{1}$ and $m_{2}$ are computed from Equation system (3.9) in terms of $\bar{X}_{f 1}$.

Plugging in all necessary constants into Equation system (3.10), after some algebraic manipulations, we obtain the following coupled equation system for the computation of $\bar{X}_{f 1}$ and $\bar{X}_{f 2}$, the optimal exercise prices in the Laplace space:

$$
\left(\begin{array}{cc}
\left(p \bar{X}_{f 1}\right)^{-\gamma_{1}} & 0 \\
0 & \left(p \bar{X}_{f 1}\right)^{-\gamma_{2}}
\end{array}\right) F_{1}\left(p \bar{X}_{f 1}\right)=\left(\begin{array}{cc}
\left(p \bar{X}_{f 2}\right)^{-\gamma_{1}} & 0 \\
0 & \left(p \bar{X}_{f 2}\right)^{-\gamma_{2}}
\end{array}\right) F_{2}\left(p \bar{X}_{f 2}\right)
$$

where

$$
\begin{aligned}
& F_{1}\left(p \bar{X}_{f 1}\right)=a_{1}+a_{2}\left(p \bar{X}_{f 1}\right), \\
& a_{1}=\frac{1}{\gamma_{2}-\gamma_{1}}\left(\begin{array}{c}
-\gamma_{2} \\
\gamma_{1}
\end{array}\right)\left(\frac{1}{p}-\psi_{2}\right) \\
& a_{2}=\frac{\bar{\psi}_{1}}{\gamma_{2}-\gamma_{1}}\left(\begin{array}{c}
\gamma_{2}-1 \\
1-\gamma_{1}
\end{array}\right)\left(\frac{1}{p}-\psi_{1}\right) \\
& F_{2}\left(p \bar{X}_{f 2}\right)=b_{1}+b_{2}\left(p \bar{X}_{f 2}\right)+b_{3}\left(\begin{array}{cc}
\left(p \bar{X}_{f 2}\right)^{k 3} & 0 \\
0 & \left(p \bar{X}_{f 2}\right)^{k 4}
\end{array}\right)\left(\begin{array}{l}
A_{3} \\
A 4
\end{array}\right) \\
& b_{1}=-\left(\begin{array}{cc}
1 & 1 \\
\gamma_{1} & \gamma_{2}
\end{array}\right)^{-1}\left[\left(\begin{array}{cc}
1 & 1 \\
k_{1} & k_{2}
\end{array}\right)\left(\begin{array}{cc}
l_{1} & l_{2} \\
l_{1} k_{1} & l_{2} k_{2}
\end{array}\right)^{-1}\left(\begin{array}{c}
\frac{r}{p(p+r)} \\
0
\end{array}\right)+\left(\begin{array}{c}
\frac{p}{p(p+r)}-\psi_{2} \\
0
\end{array}\right)\right] \\
& b_{2}=\left(\begin{array}{cc}
1 & 1 \\
\gamma_{1} & \gamma_{2}
\end{array}\right)^{-1}\left[\left(\begin{array}{cc}
1 & 1 \\
k_{1} & k_{2}
\end{array}\right)\left(\begin{array}{cc}
l_{1} & l_{2} \\
l_{1} k_{1} & l_{2} k_{2}
\end{array}\right)^{-1}\left(\begin{array}{l}
\frac{\delta}{p(p+\delta)} \\
\frac{\delta}{p(p+\delta)}
\end{array}\right)+\left(\begin{array}{l}
\frac{p}{p(p+\delta)}-\psi_{1} \\
\frac{p}{p(p+\delta)}-\psi_{1}
\end{array}\right)\right] \\
& b_{3}=-\left(\begin{array}{cc}
1 & 1 \\
\gamma_{1} & \gamma_{2}
\end{array}\right)^{-1}\left[\left(\begin{array}{cc}
1 & 1 \\
k_{1} & k_{2}
\end{array}\right)\left(\begin{array}{cc}
l_{1} & l_{2} \\
l_{1} k_{1} & l_{2} k_{2}
\end{array}\right)^{-1}\left(\begin{array}{cc}
l_{3} & l_{4} \\
l_{3} k_{3} & l_{4} k_{4}
\end{array}\right)-\left(\begin{array}{cc}
1 & 1 \\
k_{3} & k_{4}
\end{array}\right)\right]
\end{aligned}
$$


Once $A_{3}$ and $A_{4}$ are substituted in $F_{2}$, Equation system (3.11) is ready to be solved to obtain $\bar{X}_{f 1}$ and $\bar{X}_{f 2}$, the optimal exercise prices in Laplace space. This is the limit to our analytic derivation as Equation (3.11) is highly non-linear, it is not possible to obtain an explicit solution. However, the value of Equation (3.11) should not be discounted due to its complicated nature, as the ability to find the optimal exit boundary is crucial for solving an American option problem.

An appropriate numerical technique, fsolve in MATLAB, is implemented to first solve Equation (3.11) for $\bar{X}_{f 1}$ and $\bar{X}_{f 2}$ given $p$. A good choice for initial input values can speed up computation. For this purpose, we used the theoretical values of the corresponding perpetual functions and the values of the optimal exercise prices at expiry to construct a simple initial guessing function. After the values of the optimal exercise boundaries are computed, it is straightforward to calculate $\bar{U}_{1}$ and $\bar{U}_{2}$, the values of the option in Laplace space, using Equations (3.4), (3.5) and (3.6). A numerical Laplace inversion technique is then needed to obtain the values in the original time space. There are several methods for numerical inversion, the Stehfest method $(N=4$ or $N=6$ ) is used in this study due to its simplicity and reliability as pointed out in [49].

It is worth to point it out that although our approach does not produce a final analytical solution, and indeed one can solve Equation (2.4) or Equation (2.8) by some numerical method, such as FDM and FEM, our solution procedure is analytic until the final equation, (3.11). Only then numerical evaluation is needed to solve the highly non-linear coupled equations for the optimal prices in the Laplace space and to find their Laplace inversion. In fact from numerical point view, the computation involved in numerical evaluation of an analytical approach is always much less than solving PDEs directly through a numerical solution procedure, not to mention convergence and stability issues in any purely numerical approach. In addition, computation of Greeks, which are important for hedging purposes, is hard for purely numerical methods to achieve, whereas it can be easily carried out using our approach since we have analytical expressions for the option prices in the Laplace space.

\section{Numerical Examples and Discussion}

We now present numerical examples to validate our semi-analytic method described above and verify its accuracy and efficiency. In all our calculations, MATLAB 2013 and a PC with the following details were used: Intel(R) Core(TM) i7, CPU 860@2.80 GHz, 4 GB RAM, Windows 7 enterprise service pack 1, 64 bit operating system.

\subsection{Method Validation}

The results from our calculations are compared with those in the literature first to provide validation for our method. Note that we compare our results for American puts with those from others because we could not find numerical results for the call counterpart in the literature. The formulas to evaluate American put options under a regime-switching economy can be obtained in 
a similar way to those for the call counterpart in the previous section. Alternatively, the call formulas can be used to obtain the put results by using Put-Call symmetry [40]. Therefore, we will not present detailed formulation here. Two examples are discussed, one for perpetual American puts, and one for short term American puts.

\subsubsection{Perpetual American puts}

A perpetual option does not expire so its value is independent of time. As a result, the time derivative term in the governing equations (2.4) and (2.8) drops out. Close-form solutions exist for the case of perpetual American puts with regime switching [20]. Theoretically, if the expiry date $T \rightarrow \infty$ one should be able to recover the the perpetual solution from the finite expiry solution. In order to validate our formulation, we calculate values of optimal exercise prices by letting $T$ be a large number, and compare our results with those of perpetual American puts under a regime-switching economy obtained by the analytic method in [20]. For the sake of comparison, the same parameters are used. The comparison results are shown in Table 1.

Table 1: Optimal Exercise Price of Perpetual American Puts

$$
r=0.03, \delta=0, \sigma_{1}=0.9, \sigma_{2}=0.5, \lambda_{12}=\lambda_{21}=1 \text {, and } K=5
$$

\begin{tabular}{|c|c|c|c|}
\hline \multicolumn{2}{|c|}{ Analytic results [20] } & \multicolumn{2}{c|}{ Our Results } \\
\hline$S_{f 1}$ & $S_{f 2}$ & $S_{f 1}$ & $S_{f 2}$ \\
\hline 0.44055 & 0.61156 & 0.44059 & 0.61163 \\
\hline
\end{tabular}

It is evident that our results are very close to the analytical results of [20], with a maximum relative error less than $0.1 \%$. This indicates that our formula for finite expiry will degenerate to that for perpetual American options with regime-switching as $\mathrm{T}$ tends very large. We will next check if the method also performs well for evaluating American options with finite expiry dates.

\subsubsection{Finite expiry American puts}

We now compare the values of one-year American puts under a regime-switching economy in dimensional variables with those of [40]. The same parameters are used for the sake of comparison. Table 2 displays a comparison of our results with those by the Crank-Nicholson method (CN12800) and the Lattice method (LM51200) in [40].

As displayed in the table, our results compare well with the published values. Most of our results differ from the 'true' values within $1 \%$ with a maximum discrepancy of approximately $2 \%$. Our method produces more accurate results as the contract term becomes longer, and it has a maximum relative error of $0.1 \%$ for perpetual American puts. For the shorter term contracts, the efficiency in terms of our time savings far outweigh the slight differences in values. It only 
Table 2: One-year American Put Value with Regime-Switching at $t=0$ $r=0.1, \delta=0, \sigma_{1}=0.4, \sigma_{2}=0.2, \lambda_{12}=1.375968919, \lambda_{21}=1.031976689$ and $K=\$ 100$

\begin{tabular}{|c|c|c|c|c|c|c|}
\hline$S$ & \multicolumn{2}{|c|}{ CN12800 [40] } & \multicolumn{2}{c|}{ LM51200 [40] } & \multicolumn{2}{c|}{ Our Results } \\
\hline$\$$ & $V_{1}$ & $V_{2}$ & $V_{1}$ & $V_{2}$ & $V_{1}$ & $V_{2}$ \\
\hline 70 & 30.0007 & 30.0000 & 30.0006 & 30.0000 & 29.7706 & 30.0000 \\
\hline 80 & 21.2289 & 20.0000 & 21.2289 & 20.0000 & 21.0775 & 20.0000 \\
\hline 90 & 14.6191 & 11.6126 & 14.6190 & 11.6125 & 14.4933 & 11.3834 \\
\hline 100 & 9.9245 & 6.7423 & 9.9245 & 6.7423 & 9.8786 & 6.7559 \\
\hline 110 & 6.7017 & 3.9244 & 6.7017 & 3.9243 & 6.5957 & 3.8604 \\
\hline 120 & 4.5257 & 2.3083 & 4.5257 & 2.3083 & 4.4568 & 2.2928 \\
\hline 130 & 3.0665 & 1.3825 & 3.0665 & 1.3825 & 3.0488 & 1.3879 \\
\hline 140 & 2.0889 & 0.8460 & 2.0889 & 0.8460 & 2.0627 & 0.8564 \\
\hline 150 & 1.4318 & 0.5287 & 1.4318 & 0.5287 & 1.4170 & 0.5368 \\
\hline
\end{tabular}

takes maximum 0.3 seconds to obtain an option value as well as the optimal boundaries using our formula, whereas 12800 and 51200 time steps are needed to obtain the 'true' values in the Crank-Nicholson method and the Lattice method, respectively.

\subsection{Finite Expiry American calls}

In this example, we present our calculation of the optimal exercise prices and the values of American call options with finite expiry date in a regime-switching economy. The parameters used in the calculations are: $r=0.06, \delta=0.08, \sigma_{1}=0.3, \sigma_{2}=0.2, \lambda_{12}=1.375968919, \lambda_{21}=$ $1.031976689, K=\$ 100$, and $T=1$ year.

The dimensionless optimal exercise prices of one-year American call options under a regimeswitching economy are illustrated together with option values calculated under the classical BlackScholes model in Figure 4.2 with $n s$ indicating non-switching (B-S) model and rs indicating regime-switching model. As expected, the optimal exercise prices with regime-switching are bounded between those for the standard American calls; the optimal exercise price in the growth phase of the economy $\left(\sigma_{1}\right)$ is lower than that of the corresponding Black-Scholes model due to the possibility that the economy may change to the recession phase $\left(\sigma_{2}\right)$, and vice versa for the optimal exercise price in the recession phase of the economy $\left(\sigma_{2}\right)$.

The values of a one-year American call option under a regime-switching economy for various stock price $S$ are presented, and compared with those for standard American calls in Table 3. Again the results verify the theoretical analysis that the values with regime-switching should be between those of the non-switching model in [42], the values of American call under a regimeswitching economy are between those of standard American calls, that is, $V_{2}^{B S}<V_{2}<V_{1}<V_{1}^{B S}$ for $\sigma_{2}<\sigma_{1}$.

The effect of changing the dividend rate $\delta$ and the risk-free interest rate $r$ are displayed in 


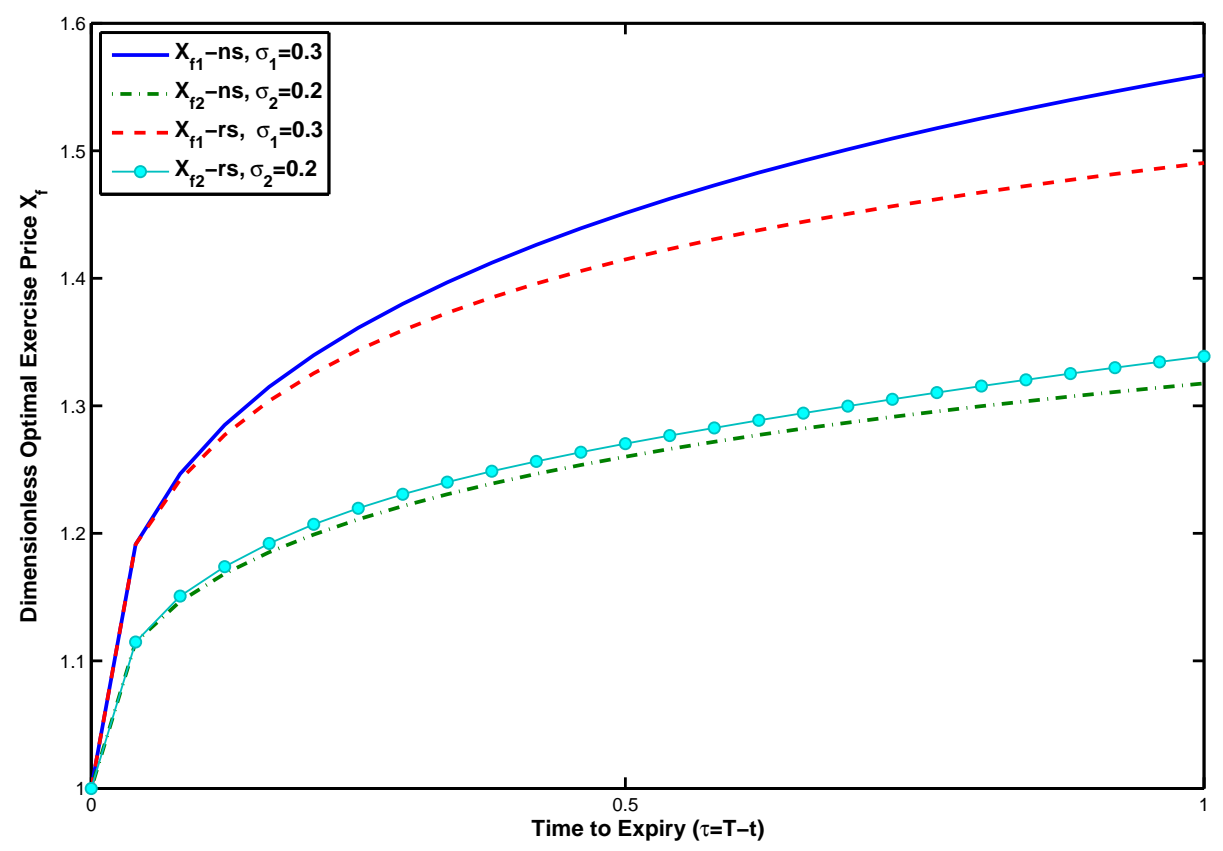

Figure 4.2: Dimensionless optimal exercise prices

Table 3: One-year American Call Option Values at $t=0$

$\quad r=0.06, \delta=0.08, \sigma_{1}=0.3, \sigma_{2}=0.2$,
$\lambda_{12}=1.375968919, \lambda_{21}=1.031976689$ and $K=100$
\begin{tabular}{|c|c|c|c|c|}
\hline$S$ & \multicolumn{2}{|c|}{ Our results } & \multicolumn{2}{c|}{ BS } \\
\cline { 2 - 5 } & $V_{1}$ & $V_{2}$ & $V_{1}$ & $V_{2}$ \\
\hline 80 & 2.264831 & 1.47024 & 2.9753 & 0.91842 \\
\hline 100 & 9.351436 & 7.78078 & 10.465 & 6.7532 \\
\hline 120 & 22.50430 & 20.71355 & 23.143 & 20.288 \\
\hline
\end{tabular}

Figure 4.3 and Figure 4.4, respectively. The sensitivity of the key parameters follows the same trend as that of an American call option in a non-switching economy.

\section{Concluding remarks}

In this paper, we develop a modified semi-analytical method to evaluate American options under s two-state regime-switching economy. Numerical examples for American options in finite and infinite maturity are presented, and the results compare well with those of existing methods. Further, the results show that our method provides an efficient as well as accurate valuation, 


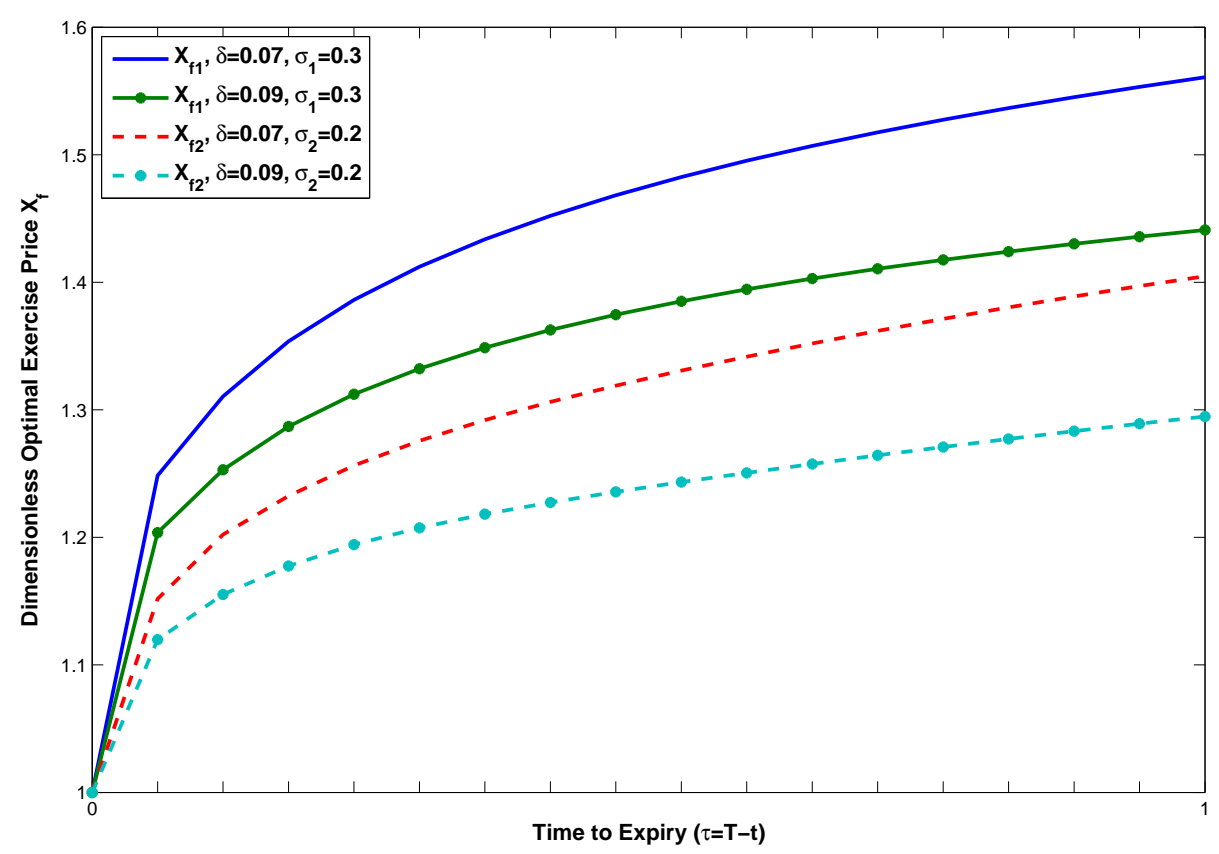

Figure 4.3: Dimensionless optimal exercise prices for different dividend rate

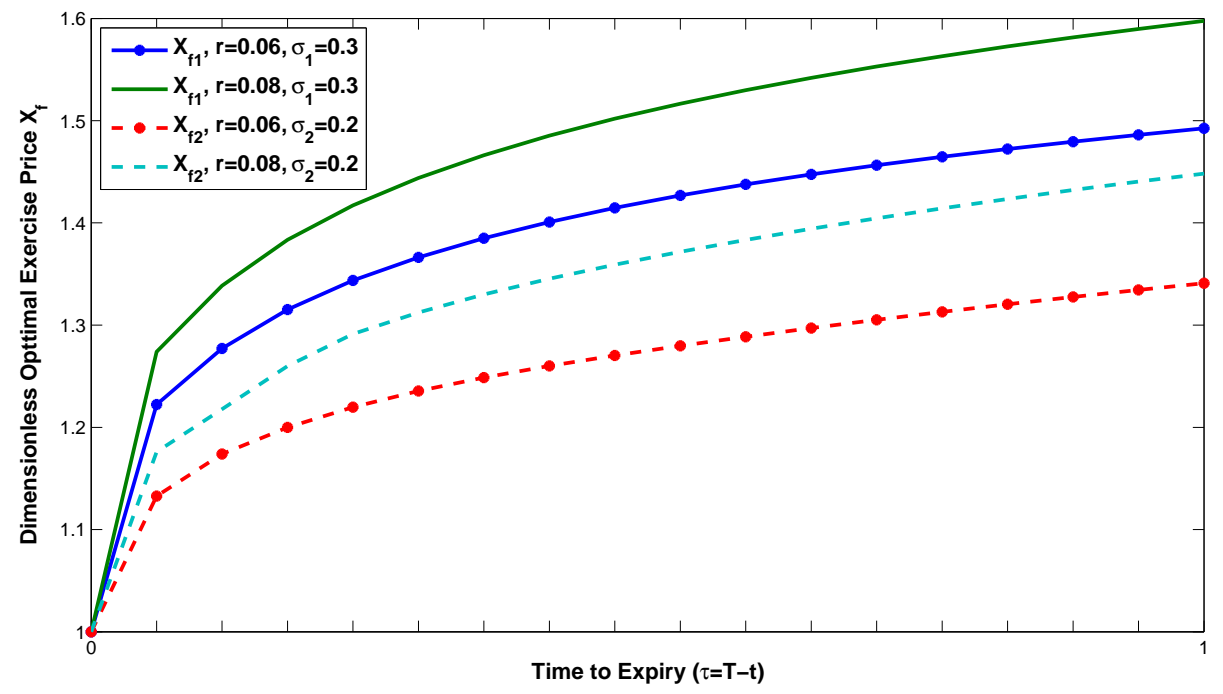

Figure 4.4: Dimensionless optimal exercise prices for different interest rate

especially for longer term contracts.

The approach could be adopted to the evaluation of American barrier options under a regimeswitching economy. The easiest example is American down-and-out options, only the lower boundary condition needs to be changed, that is, homogeneous boundary conditions at the barrier instead of at the zero stock value. The derivation of the final equations would be similar, but 
more tedious, as we have two extra constants to be decided. However, for other types of barrier options, it is not straight forward. Indeed it is part of our plan for future research.

\section{Appendix A Solution for Common Continuation Region}

In this appendix the PDE systems in equation (3.1) are solved by following the technique in [46].

Employing two intervals in the common continuation region, $1<X \leq X_{f 2}$ and $X \leq 1$, the PDE system for $U_{1}$ in equation (3.1) can be presented as follows:

$$
\begin{cases}-\frac{\partial U_{1}}{\partial \tau}+\frac{1}{2} \sigma_{1}^{2} X^{2} \frac{\partial^{2} U_{1}}{\partial X^{2}}+(r-\delta) X \frac{\partial U_{1}}{\partial X}-r U_{1}=\lambda_{12}\left(U_{1}-U_{2}\right), & \text { if } 1<X \leq X_{f 2} \\ U_{1}(0, \tau)=0 & \\ U_{1}(X, 0)=X-1 & \\ -\frac{\partial U_{1}}{\partial \tau}+\frac{1}{2} \sigma_{1}^{2} X^{2} \frac{\partial^{2} U_{1}}{\partial X^{2}}+(r-\delta) X \frac{\partial U_{1}}{\partial X}-r U_{1}=\lambda_{12}\left(U_{1}-U_{2}\right), & \text { if } X \leq 1 ; \\ U_{1}(0, \tau)=0 & \\ U_{1}(X, 0)=0 & \end{cases}
$$

The following continuity conditions are enforced to ensure that $U_{1}$ is $C^{1}$ continuous at $X=1$ :

$$
\left\{\begin{array}{l}
\lim _{x \rightarrow 1^{-}} U_{1}=\lim _{x \rightarrow 1^{+}} U_{1} \\
\lim _{x \rightarrow 1^{-}} \frac{\partial U_{1}}{\partial X}=\lim _{x \rightarrow 1^{+}} \frac{\partial U_{1}}{\partial X}
\end{array}\right.
$$

Similarly, the PDE system for $U_{2}$ becomes:

$$
\begin{cases}-\frac{\partial U_{2}}{\partial \tau}+\frac{1}{2} \sigma_{2}^{2} X^{2} \frac{\partial^{2} U_{2}}{\partial X^{2}}+(r-\delta) X \frac{\partial U_{2}}{\partial X}-r U_{2}=\lambda_{21}\left(U_{2}-U_{1}\right), & \text { if } 1<X \leq X_{f 2} \\ U_{2}(0, \tau)=0 & \\ U_{2}(X, 0)=X-1 & \\ U_{2}\left(X_{f 2}, \tau\right)=X_{f 2}-1 & \\ \frac{\partial U_{2}}{\partial X}\left(X_{f 2}, \tau\right)=1 & \text { if } X \leq 1 \\ -\frac{\partial U_{2}}{\partial \tau}+\frac{1}{2} \sigma_{2}^{2} X^{2} \frac{\partial^{2} U_{2}}{\partial X^{2}}+(r-\delta) X \frac{\partial U_{2}}{\partial X}-r U_{2}=\lambda_{21}\left(U_{2}-U_{1}\right), & \\ U_{2}(0, \tau)=0 & \\ U_{2}(X, 0)=0 & \end{cases}
$$

with the continuity conditions at $X=1$ : 


$$
\left\{\begin{array}{l}
\lim _{x \rightarrow 1^{-}} U_{2}=\lim _{x \rightarrow 1^{+}} U_{2} \\
\lim _{x \rightarrow 1^{-}} \frac{\partial U_{2}}{\partial X}=\lim _{x \rightarrow 1^{+}} \frac{\partial U_{2}}{\partial X}
\end{array}\right.
$$

Subsequently, equations (A.1 - A.4) are transformed to the Laplace space as follows

$$
\begin{aligned}
& \begin{cases}\frac{1}{2} \sigma_{1}^{2} X^{2} \frac{d^{2} \bar{U}_{1}}{d X^{2}}+(r-\delta) X \frac{d \bar{U}_{1}}{d X}-\left(p+r+\lambda_{12}\right) \bar{U}_{1}+\lambda_{12} \bar{U}_{2}=-(X-1), & \text { if } 1<X \leq X_{f 1} \\
\bar{U}_{1}(0, p)=0 & \text { if } X \leq 1 ; \\
\frac{1}{2} \sigma_{1}^{2} X^{2} \frac{d^{2} \bar{U}_{1}}{d X^{2}}+(r-\delta) X \frac{d \bar{U}_{1}}{d X}-\left(p+r+\lambda_{12}\right) \bar{U}_{1}+\lambda_{12} \bar{U}_{2}=0, & \\
\bar{U}_{1}(0, p)=0 & \end{cases} \\
& \left\{\begin{array}{l}
\bar{U}_{1}\left(1^{-}, p\right)=\bar{U}_{1}\left(1^{+}, p\right) \\
\frac{d \bar{U}_{1}}{d X}\left(1^{-}, p\right)=\frac{d \bar{U}_{1}}{d X}\left(1^{+}, p\right)
\end{array}\right. \\
& \begin{cases}\frac{1}{2} \sigma_{2}^{2} X^{2} \frac{d^{2} \bar{U}_{2}}{d X^{2}}+(r-\delta) X \frac{d \bar{U}_{2}}{d X}-\left(p+r+\lambda_{21}\right) \bar{U}_{2}+\lambda_{21} \bar{U}_{1}=-(X-1), & \text { if } 1<X \leq X_{f 2} ; \\
\bar{U}_{2}(0, p)=0 & \\
\bar{U}_{2}\left(p \bar{X}_{f 2}, p\right)=\bar{X}_{f 2}-\frac{1}{p} & \\
\frac{d U_{2}}{d X}\left(p \bar{X}_{f 2}, p\right)=\frac{1}{p} & \text { if } X \leq 1 ; \\
\frac{1}{2} \sigma_{2}^{2} X^{2} \frac{d^{2} \bar{U}_{2}}{d X^{2}}+(r-\delta) X \frac{d \bar{U}_{2}}{d X}-\left(p+r+\lambda_{21}\right) \bar{U}_{2}+\lambda_{21} \bar{U}_{1}=0, & \\
\bar{U}_{2}(0, p)=0 & \end{cases} \\
& \left\{\begin{array}{l}
\bar{U}_{2}\left(1^{-}, p\right)=\bar{U}_{2}\left(1^{+}, p\right) \\
\frac{d \bar{U}_{2}}{d X}\left(1^{-}, p\right)=\frac{d \bar{U}_{2}}{d X}\left(1^{+}, p\right)
\end{array}\right.
\end{aligned}
$$

Equations (A.5 - A.8) are ODE systems which are easier to be solved. In performing the Laplace transform, the pseudo-steady-state approximation proposed by Zhu [46] is applied to the free boundary.

The solutions to the ODE systems are in the following form:

$$
\bar{U}_{1}(X, p)= \begin{cases}A_{1} X^{k_{1}}+A_{2} X^{k_{2}}+A_{3} X^{k_{3}}+A_{4} X^{k_{4}}+A A(X, p), & \text { if } 1<X \leq X_{f 2} \\ A_{5} X^{k_{1}}+A_{6} X^{k_{2}}+A_{7} X^{k_{3}}+A_{8} X^{k_{4}}, & \text { if } X \leq 1\end{cases}
$$




$$
\bar{U}_{2}(X, p)= \begin{cases}B_{1} X^{k_{1}}+B_{2} X^{k_{2}}+B_{3} X^{k_{3}}+B_{4} X^{k_{4}}+B B(X, p), & \text { if } 1<X \leq X_{f 2} \\ B_{5} X^{k_{1}}+B_{6} X^{k_{2}}+B_{7} X^{k_{3}}+B_{8} X^{k_{4}}, & \text { if } X \leq 1 ;\end{cases}
$$

where

$$
A A(X, p)=B B(X, p)=\frac{1}{p+\delta} X-\frac{1}{p+r} .
$$

The constants $A_{i}$ and $B_{i}$ are related by the following equation:

$$
\left\{\frac{1}{2} \sigma_{1}^{2} k_{i}\left(k_{i}-1\right)+(r-\delta) k_{i}-\left(p+r+\lambda_{12}\right)\right\} A_{i}+\lambda_{12} B_{i}=0
$$

or

$$
\left\{\frac{1}{2} \sigma_{2}^{2} k_{i}\left(k_{i}-1\right)+(r-\delta) k_{i}-\left(p+r+\lambda_{21}\right)\right\} B_{i}+\lambda_{21} A_{i}=0
$$

where $k_{i}, i=1 \ldots 4$ are the solutions to the quartic indicial equation (C.2). Further, in Appendix $\mathrm{C}$ we proved that

$$
k_{1}<k_{2}<0<k_{3}<k_{4} \text {. }
$$

Recall the boundary condition $\bar{U}_{1,2}(0, p)=0$, it is apparent that the solutions in the region $X<1$ can not involve negative powers of $X$. This means that the coefficients of $X^{k_{1}}$ and $X^{k_{2}}$ in the solutions for $X<1$ should be zero, that is, $A_{5}=A_{6}=B_{5}=B_{6}=0$. Renaming the constants $A_{7}, A_{8}$ and $B_{7}, B_{8}$ to $A_{5}, A_{6}$ and $B_{5}, B_{6}$, we obtain the final solutions of $\bar{U}_{1}$ and $\bar{U}_{2}$ in the Laplace space:

$$
\begin{aligned}
& \bar{U}_{1}(X, p)= \begin{cases}A_{1} X^{k_{1}}+A_{2} X^{k_{2}}+A_{3} X^{k_{3}}+A_{4} X^{k_{4}}+A A(X, p), & \text { if } 1<X \leq X_{f 2} ; \\
A_{5} X^{k_{3}}+A_{6} X^{k_{4}}, & \text { if } X \leq 1 ;\end{cases} \\
& \bar{U}_{2}(X, p)= \begin{cases}B_{1} X^{k_{1}}+B_{2} X^{k_{2}}+B_{3} X^{k_{3}}+B_{4} X^{k_{4}}+B B(X, p), & \text { if } 1<X \leq X_{f 2} ; \\
B_{5} X^{k_{3}}+B_{6} X^{k_{4}}, & \text { if } X \leq 1 ;\end{cases}
\end{aligned}
$$

The fact that $A_{i}$ are related to $B_{i}$ allows us to solve only for the $A_{i}$ 's in the final equations. 


\section{Appendix B Solution for Transition Region}

In this appendix, we present the steps to solve the PDE system in transition region. We start by transforming the equation system (3.2) into the Laplace space:

$$
\left\{\begin{array}{l}
\frac{1}{2} \sigma_{1}^{2} X^{2} \frac{d^{2} \bar{U}_{1}}{d X^{2}}+(r-\delta) X \frac{d \bar{U}_{1}}{d X}-\left(p+r+\lambda_{12}\right) \bar{U}_{1}=-(X-1)-\frac{\lambda_{12}(X-1)}{p} \\
\bar{U}_{1}\left(p \bar{X}_{f 1}, p\right)=\bar{X}_{f 1}-\frac{1}{p} \\
\frac{d \bar{U}_{1}}{d X}\left(p \bar{X}_{f 1}, p\right)=\frac{1}{p}
\end{array}\right.
$$

The solution of equation (B.1) has a general form of

$$
\bar{U}_{1}=m_{1} X^{\gamma_{1}}+m_{2} X^{\gamma_{2}}+M(X, p)
$$

where $m_{1,2}$ are integration constants, $\gamma_{1,2}$ are the solutions of the indicial equation

$$
\frac{1}{2} \sigma_{1}^{2} \gamma^{2}+\left(r-\delta-\frac{1}{2} \sigma_{1}^{2}\right) \gamma-\left(p+r+\lambda_{12}\right)=0,
$$

and $M(X, p)$ is the particular solution of equation (B.1),

$$
M(X, p)=\left\{\frac{p+\lambda_{12}}{p\left(p+\delta+\lambda_{12}\right)}\right\} X-\left\{\frac{p+\lambda_{12}}{p\left(p+r+\lambda_{12}\right)}\right\}
$$

or we can write as follows:

$$
M(X, p)=\psi_{1} X-\psi_{2}
$$

where

$$
\psi_{1}=\left\{\frac{p+\lambda_{12}}{p\left(p+\delta+\lambda_{12}\right)}\right\}
$$

and

$$
\psi_{2}=\left\{\frac{p+\lambda_{12}}{p\left(p+r+\lambda_{12}\right)}\right\}
$$




\section{Appendix C Solution of Indicial Equations}

The solutions in the Laplace space in the common continuation region are presented in equations (A.9) and (A.10). Substituting these solutions and their derivatives into equations (A.5) and (A.7), we obtain the relation between constants $A_{i}$ and $B_{i}$ :

$$
\left\{\frac{1}{2} \sigma_{1}^{2} k_{i}\left(k_{i}-1\right)+(r-\delta) k_{i}-\left(p+r+\lambda_{12}\right)\right\} A_{i}+\lambda_{12} B_{i}=0
$$

or

$$
\left\{\frac{1}{2} \sigma_{2}^{2} k_{i}\left(k_{i}-1\right)+(r-\delta) k_{i}-\left(p+r+\lambda_{21}\right)\right\} B_{i}+\lambda_{21} A_{i}=0
$$

The last two equations are equivalent. Let $l\left(k_{i}\right)=\frac{1}{2} \sigma_{1}^{2} k_{i}\left(k_{i}-1\right)+(r-\delta) k_{i}-\left(p+r+\lambda_{12}\right)$, the relation between $A_{i}$ and $B_{i}$ can be expressed as follows

$$
B_{i}=-A_{i} \frac{l\left(k_{i}\right)}{\lambda_{12}}, \quad \text { for } \quad i=1 \ldots 4 \quad \text { and } \quad B_{i}=-A_{i} \frac{l\left(k_{i-4}\right)}{\lambda_{12}}, \quad \text { for } \quad i=5 \ldots 8
$$

Note that for simplicity we use $l_{i}=l\left(k_{i}\right), \quad i=1,2,3,4$ in the main sections of this paper.

In common continuation region, the indicial equation for the corresponding differential equations is a quartic equation as written below:

$$
F(k)=l(k) g(k)-\lambda_{12} \lambda_{21}
$$

where $l(k)$ and $g(k)$ are quadratic functions

$$
l(k)=\frac{1}{2} \sigma_{1}^{2} k^{2}+\left(r-\delta-\frac{1}{2} \sigma_{1}^{2}\right) k-\left(p+r+\lambda_{12}\right)
$$

and

$$
g(k)=\frac{1}{2} \sigma_{2}^{2} k^{2}+\left(r-\delta-\frac{1}{2} \sigma_{2}^{2}\right) k-\left(p+r+\lambda_{21}\right)
$$

Let

$$
l(k)=\frac{1}{2} \sigma_{1}^{2} k^{2}+\left(r-\delta-\frac{1}{2} \sigma_{1}^{2}\right) k-\left(p+r+\lambda_{12}\right)=0 .
$$

Since $\lim _{k \rightarrow \infty} l(k)=\lim _{k \rightarrow-\infty} l(k) \rightarrow \infty$ and $l(0)=-\left(p+r+\lambda_{12}\right)<0 \quad\left(p, r, \lambda_{12}>0\right)$, it can be concluded that Equation (C.3) has two real roots, say, $k k_{1}<0$ and $k k_{2}>0$.

Now, $F\left(k k_{1}\right)=F\left(k k_{2}\right)=-\lambda_{12} \lambda_{21}<0\left(\lambda_{12}, \lambda_{21}>0\right), F(0)=(p+r)\left(p+r+\lambda_{12}+\lambda_{21}\right)>0$ and $\lim _{k \rightarrow \infty} F(k)=\lim _{k \rightarrow-\infty} F(k) \rightarrow \infty$. Hence, we can conclude that the quartic function $F(k)$ has four real roots $k_{1}, k_{2}, k_{3}, k_{4}$, two of which are positive and two negative, that is,

$$
k_{1}<k_{2}<0<k_{3}<k_{4}
$$




\section{Appendix D List of Integration Constants}

Here we list the final result for the integration constants in Section 3 without the tedious algebraic details:

$$
\begin{aligned}
& \left(\begin{array}{l}
A_{1} \\
A_{2}
\end{array}\right)=\left[\left(\begin{array}{cc}
1 & 1 \\
k_{3} & k_{4}
\end{array}\right)^{-1}\left(\begin{array}{cc}
1 & 1 \\
k_{1} & k_{2}
\end{array}\right)-\left(\begin{array}{cc}
l_{3} & l_{4} \\
l_{3} k_{3} & l_{4} k_{4}
\end{array}\right)^{-1}\left(\begin{array}{cc}
l_{1} & l_{2} \\
l_{1} k_{1} & l_{2} k_{2}
\end{array}\right)\right]^{-1} \\
& {\left[\left(\begin{array}{cc}
1 & 1 \\
k_{3} & k_{4}
\end{array}\right)^{-1}-\left(\begin{array}{cc}
l_{3} & l_{4} \\
l_{3} k_{3} & l_{4} k_{4}
\end{array}\right)^{-1}\right]\left(\begin{array}{c}
\frac{1}{p+r}-\frac{1}{p+\delta} \\
-\frac{1}{p+\delta}
\end{array}\right)} \\
& \left(\begin{array}{l}
A_{3} \\
A_{4}
\end{array}\right)=\left(\begin{array}{cc}
\left(p \bar{X}_{f 2}\right)^{-k_{3}} & 0 \\
0 & \left(p \bar{X}_{f 2}\right)^{-k_{4}}
\end{array}\right)\left(\begin{array}{cc}
l_{3} & l_{4} \\
l_{3} k_{3} & l_{4} k_{4}
\end{array}\right)^{-1} \\
& {\left[\left(\begin{array}{c}
\frac{\delta}{p(p+\delta)}\left(p \bar{X}_{f 2}\right)-\frac{r}{p(p+r)} \\
\frac{\delta}{p(p+\delta)}\left(p \bar{X}_{f 2}\right)
\end{array}\right)-\left(\begin{array}{cc}
l_{1} & l_{2} \\
l_{1} k_{1} & l_{2} k_{2}
\end{array}\right)\left(\begin{array}{cc}
\left(p \bar{X}_{f 2}\right)^{k_{1}} & 0 \\
0 & \left(p \bar{X}_{f 2}\right)^{k_{2}}
\end{array}\right)\right.} \\
& {\left[\left(\begin{array}{cc}
1 & 1 \\
k_{3} & k_{4}
\end{array}\right)^{-1}\left(\begin{array}{cc}
1 & 1 \\
k_{1} & k_{2}
\end{array}\right)-\left(\begin{array}{cc}
l_{3} & l_{4} \\
l_{3} k_{3} & l_{4} k_{4}
\end{array}\right)^{-1}\left(\begin{array}{cc}
l_{1} & l_{2} \\
l_{1} k_{1} & l_{2} k_{2}
\end{array}\right)\right]^{-1}} \\
& \left.\left(\begin{array}{cc}
1 & 1 \\
k_{3} & k_{4}
\end{array}\right)^{-1}\left(\begin{array}{cc}
l_{3} & l_{4} \\
l_{3} k_{3} & l_{4} k_{4}
\end{array}\right)^{-1}\left(\begin{array}{c}
\frac{1}{p+r}-\frac{1}{p+\delta} \\
-\frac{1}{p+\delta}
\end{array}\right)\right] \\
& \left(\begin{array}{l}
A_{5} \\
A_{6}
\end{array}\right)=\left(\begin{array}{cc}
1 & 1 \\
k_{3} & k_{4}
\end{array}\right)^{-1}\left[\left(\begin{array}{cc}
1 & 1 \\
k_{1} & k_{2}
\end{array}\right)\left(\begin{array}{l}
A_{1} \\
A_{2}
\end{array}\right)+\left(\begin{array}{cc}
1 & 1 \\
k_{3} & k_{4}
\end{array}\right)\left(\begin{array}{l}
A_{3} \\
A_{4}
\end{array}\right)-\left(\begin{array}{c}
\frac{1}{p+r}-\frac{1}{p+\delta} \\
-\frac{1}{p+\delta}
\end{array}\right)\right] \\
& \left(\begin{array}{c}
m_{1} \\
m_{2}
\end{array}\right)=\left(\begin{array}{cc}
1 & 1 \\
\gamma_{1} & \gamma_{2}
\end{array}\right)^{-1}\left(\begin{array}{cc}
\left(p \bar{X}_{f 1}\right)^{\gamma_{1}} & 0 \\
0 & \left(p \bar{X}_{f 1}\right)^{\gamma_{2}}
\end{array}\right)\left(\begin{array}{c}
p \bar{X}_{f 1}-1 \\
p \bar{X}_{f 1}
\end{array}\right)\left(\frac{1}{p}-\psi_{2}\right)
\end{aligned}
$$

\section{References}

[1] Alexander, C., And Nogueira, L. M. Hedging with stochastic and local volatility. Working Paper ISMA Centre, The University of Reading (2004). 
[2] Avellaneda, M., Levy, A., Pars, A., and Par'as, A. Pricing and hedging derivative securities in markets with uncertain volatilities. Applied Mathematical Finance 2 (1995), $73-88$.

[3] Avellaneda, M. and Buff, R. Combinatorial implications of nonlinear uncertain volatility models: the case of barrier options. Applied Mathematical Finance 6, 1 (1999), 1-18.

[4] Ball, C. A., And Roma, A. Stochastic volatility option pricing. Journal of Financial and Quantitative Analysis 29 (12 1994), 589-607.

[5] Bansal, R., and Zhou, H. Term structure of interest rates with regime shifts. The Journal of Finance 57, 5 (2002), 1997-2043.

[6] Barone-Adesi, G., And Whaley, R. E. Efficient analytic approximation of American option values. Journal of Finance 42 (1987), 301-320.

[7] Beetsma, R., And Giuliodori, M. The changing macroeconomic response to stock market volatility shocks. Journal of Macroeconomics 34, 2 (2012), 281 - 293.

[8] Bernard Dumas, J. F., And Whaley, R. E. Implied volatility functions: Empirical tests. The Journal of Finance L111, 6 (1998), 2059-2106.

[9] Boyle, P., And Draviam, T. Pricing exotic options under regime-switching. Insurance: Mathematics and Economics 40, 2 (2007), $267-282$.

[10] Buffington, J., And Elliott, R. J. American options with regime-switching. International Journal of Theoretical and Applied Finance 05, 05 (2002), 497-514.

[11] Buffington, J., And Elliott, R. J. Regime-switching and European options. Stochastic Theory and Control Lecture Notes in Control and Information Sciences 280 (2002), 382.

[12] Carr, P., And Madan, D. B. Option valuation using the fast Fourier transform. Journal of Computational Finance 2 (1999), 61-73.

[13] Carr, P., And Sun, J. A new approach for option pricing under stochastic volatility. Review of Derivatives Research 10, 2 (2007), 87-150.

[14] Chen, C.-C., And Hung, M.-Y. Option pricing under Markov-switching GARCH processes. Journal of Futures Markets 30, 5 (2010), 444-464.

[15] Derman, E., And Kani, I. The volatility smile and its implied tree. Tech. rep., Goldman Sachs, 1994. Quantitative Strategies Research Notes.

[16] Dupire, B. Pricing with a smile. Risk Magazine \%, 1 (1994), 18-20.

[17] Engelmann, B., Fengler, M. R., Nalholm, M., and Schwendner, P. Static versus dynamic hedges: an empirical comparison for barrier options. Review of Derivatives Research 9, 3 (2006), 239-264. 
[18] French, K. R., G.Schwert, W., and Stambaugh, R. F. Expected stock returns and volatility. Journal of Financial Economics 19 (1987), 3-29.

[19] Guo, X. Inside information and stock fluctuations, 1999. Copyright UMI - Dissertations Publishing 1999 .

[20] Guo, X., And Zhang, Q. Closed-form solutions for perpetual American put options with regime-switching. SIAM Journal on Applied Mathematics 64, 6 (2004), 2034-2049.

[21] Guo, X., And Zhang, Q. Optimal selling rules in a regime-switching model. IEEE Transactions on Automatic Control 9, 50 (2007), 1450-1455.

[22] Hardy, M. R. A regime-switching model of long-term stock returns. North American Actuarial Journal 5, 2 (2001), 41-53.

[23] Heston, S. L. A closed-form solution for options with stochastic volatility with applications to bond and currency options. The Review of Financial Studies 6, 2 (1993), 327-343.

[24] Holmes, A. D., Yang, H., And Zhang, S. A front-fixing finite element method for the valuation of American options with regime-switching. International Journal of Computer Mathematics 89, 9 (2012), 1094-1111.

[25] Huang, Y., Forsyth, P. A., and Labahn, G. Methods for pricing American options under regime-switching. SIAM Journal on Scientific Computing 33, 5 (2011), 2144-25.

[26] Hull, J., And White, A. The pricing of options on assets with stochastic volatilities. The Journal of Finance 42, 2 (1987), 281-300.

[27] Hull, J. C. Options, futures, and other derivatives, 7th edition ed. Pearson Prentice Hall, 2009.

[28] Kahl, C., And JaCkel, P. Not-so-complex logarithms in the Heston model. Working paper, 2006.

[29] Khaliq, A. Q. M., And Liu, R. New numerical scheme for pricing American options with regime-switching. International Journal of Theoretical and Applied Finance 12 (2009), 319-340.

[30] Mamon, R. S., And Rodrigo, M. R. Explicit solutions to European options in a regimeswitching economy. Operations Research Letters 33, 6 (2005), 581 - 586.

[31] NAIK, V. Option valuation and hedging strategies with jumps in the volatility of asset returns. The Journal of Finance 48, 5 (1993), pp. 1969-1984.

[32] Patrick S. Hagan, Deep Kumar, A. S. L. A. D. E. W. Managing smile risk. Wilmott Magazine 1 (2002), 84-108. 
[33] Sabanis, S. Stochastic volatility. International Journal of Theoretical and Applied Finance $5,5(2002), 515$.

[34] Schwert, G. W. Why does stock market volatility change over time? The Journal of Finance 44, 5 (1989), pp. 1115-1153.

[35] Scruggs, J. T., And NArdari, F. Why does stock market volatility change over time? a time-varying variance decomposition for stock returns. EFA 2005 Moscow Meetings, August 2005 .

[36] Shiller, R. J. Causes of changing financial market volatility. Financial Market Volatility (1989), 1-22.

[37] Stehfest, H. Algorithm 368: numerical inversion of Laplace transforms [D5]. Commun. ACM 13, 1 (Jan. 1970), 47-49.

[38] Stein, E. M., And Stein, J. C. Stock price distributions with stochastic volatility: an analytic approach. The Review of Financial Studies 4, 4, 727.

[39] Thavaneswaran, A., Singh, J., and Appadoo, S. Option pricing for some stochastic volatility models. The Journal of Risk Finance 7, 4 (2006), 425-445.

[40] YAng, H. A numerical analysis of American options with regime-switching. Journal of Scientific Computing 44 (2010), 69-91.

[41] Yao, D., Zhang, Q., And Zhou, X. Y. A regime-switching model for European options. In Stochastic processes, optimization, and control theory: applications in financial engineering, queueing networks, and manufacturing systems, H. Yan, G. Yin, and Q. Zhang, Eds., vol. 94 of International Series in Operations Research $\&$ Management Science. Springer US, 2006, pp. $281-300$.

[42] YI, F. American put option with regime-switching volatility (finite time horizon)variational inequality approach. Mathematical Methods in the Applied Sciences 31, 12 (2008), 1461-1477.

[43] Yin, G., Wang, J. W., And Zhang, Q. A new approach for pricing American put options. In Decision and Control, 2004. CDC. 43rd IEEE Conference on (2004), vol. 4, pp. 3925-3929 Vol.4.

[44] Yuen, F. L., AND YANG, H. Option pricing with regime-switching by trinomial tree method. Journal of Computational and Applied Mathematics 233, 8 (2010), 1821 - 1833.

[45] Zhang, K., Teo, K., And Swartz, M. A robust numerical scheme for pricing American options under regime-switching based on penalty method. Computational Economics 43, 4 (2014), 463-483. 
[46] ZHU, S.-P. A new analytical approximation formula for the optimal exercise boundary of American put options. International Journal of Theoretical and Applied Finance 7, 9 (2006), $1141-1177$.

[47] Zhu, S.-P., Badran, A., And Lu, X. A new exact solution for pricing European options in a two-state regime-switching economy. Computers 83 Mathematics with Applications 64, 8 (2012), $2744-2755$.

[48] Zhu, S.-P., And Chen, W.-T. A new analytical approximation for European puts with stochastic volatility . Applied Mathematics Letters 23, 6 (2010), 687 - 692.

[49] Zhu, S.-P., And Zhang, J. Using Laplace transform to price American puts. Dynamics of Continuous, Discrete and Impulsive Systems-B:Applications and Algorithms 19, 4 (2012), 447-469.

[50] Zhylyevskyy, O. Efficient pricing of European-style options under Hestons stochastic volatility model. Theoretical Economics Letters (2012). 